\title{
A Bayesian Approach to Block Structure Inference in AV1-based Multi-rate Video Encoding
}

\author{
Bichuan Guo*, Xinyao Chen*, Jiawen Gu*, Yuxing $\operatorname{Han}^{\dagger}$, Jiangtao Wen* \\ *Tsinghua Univeristy \\ †South China Agricultural Univeristy \\ Beijing, 100084, China \\ jtwen@tsinghua.edu.cn \\ Guangzhou, Guangdong, 510642, China \\ yuxinghan@scau.edu.cn
}

\begin{abstract}
Due to differences in frame structure, existing multi-rate video encoding algorithms cannot be directly adapted to encoders utilizing special reference frames such as AV1 without introducing substantial rate-distortion loss. To tackle this problem, we propose a novel bayesian block structure inference model inspired by a modification to an HEVC-based algorithm. It estimates the posterior probabilistic distributions of block partitioning, and adapts early terminations in the RDO procedure accordingly. Experimental results show that the proposed method provides flexibility for controlling the tradeoff between speed and coding efficiency, and can achieve an average time saving of $36.1 \%$ (up to 50.6\%) with negligible bitrate cost.
\end{abstract}

\section{Introduction}

Video content accounts for the majority of all internet traffic, and has been growing steadily [1]. Due to the dynamic nature of public network conditions, adaptive streaming is widely used by video content providers [2], where the same video is encoded to different rates (and correspondingly qualities, denote as a variable $Q$ ), with a client-side algorithm adaptively requests a version based on network conditions.

On the other hand, AOMedia Video 1 (AV1 for short) is an open, royalty-free video coding format developed by the Alliance for Open Media [3][4]. Based on its predecessor VP9, AV1 utilizes numerous new coding tools to achieve cutting-edge coding efficiency. As the coding complexity increases, real time encoding becomes a challenge for adaptive live streaming, and it is thus of great interest to reduce the overall complexity without substantial degradation to the coding efficiency.

In addition to the challenges inherent in real time encoding a single AV1 stream, encoding an input video to multiple rates in parallel is another challenge of great interests to adaptive video streaming. Given that the rate-distortion optimizations (RDO) in multiple encodings of the same video is correlated, parameters and intermediate results from the different RDO procedures can be shared among the processes. Such parameters and intermediate results include prediction modes, motion vectors/intra modes, and block structures [5]. It has been shown [5][6][7] that the encoder complexity can be significantly reduced by considering block structures alone.

In this paper, we propose a bayesian inference model for block structures in video codecs using special long-term reference frames. It exploits the statistical correlation between encoding processes for the same video input with different target quality levels and identical input/output spatial resolutions. The AV1 codec is used as a 
benchmark tool to demonstrate the effectiveness of our model without loss of generality, since the proposed model can be applied to other codecs of similar principles.

The rest of the paper is organized as follows. Related work is presented in Section II. A study on the statistical behavior of block structure decisions of the AV1 codec is given in Section III. Adaptation of an existing algorithm from HEVC to AV1 and its improvement are discussed in Section IV, based on which, a detailed description and analysis of our proposed bayesian inference model are given in Section V, followed by experimental results in Section VI. Finally, Section VII concludes the paper.

\section{Related Work}

Many efforts have been dedicated to reduce the complexity of video encoding. [8][9] proposed a wavefront parallel processing method that exploits spatial independencies in H.264/AVC, which have since been adopted by the x264 open source H.264 encoder [10]. [11] improves it by jointly considering temporal independencies. These methods accelerate video encoding on multi-core systems without reducing its complexity. Another approach [12][13] is to use transcoding [14] for multiple encoding of the same input. Because the RDO mode decisions and motion estimation processes are bitrate dependent, and different video coding formats such as H.264 and H.265 are very different, transcoding by simply re-quantizating the residual calculated using the mode and motion information obtained from the RDO for a encoding format and a different rate may introduce significant quality losses, both from not fully utilizing encoding tools in a target format, and from sub-optimal RDO decisions.

Simultaneous encoding of the same input, also called multi-rate encoding [15], reduces the overall complexity while retaining high fidelity. The encoder performs full encoding on a chosen instance as a reference instance, while other instances (referred to as local instances) consult the reference instance to infer their optimal RDO decisions. [16] proposed a preliminary framework which merely copies decisions from the reference instance, resulting in considerable rate-distortion (RD) loss. [5][7] refined this framework by searching in a pruned RDO recursion tree, reducing the RD loss to a negligible level. However, as shown later in this paper, this method cannot be directly migrated to AV1 without introducing substantial performance degradation. [6] proposed an ensemble learning method to predict optimal block structures in HEVC multi-rate encoding.

Bayesian inference is widely used in video encoding. [17][18] used bayesian models to accelerate skip mode and CU size decisions in HEVC. [19] uses a bayesian model to accelerate prediction mode decisions in x265 multi-rate encoding. There has been very little reported work that has considered block structure decisions with RDO of both the reference instance and the local instance in multi-rate encoding.

As its main contribution, the current paper proposes a joint probabilistic model to infer optimal block structure decisions. For a particular local instance, its past block structure decisions will be recorded to update the inference model, which in turn computes the probabilistic distribution of its optimal block structure decisions with the aid of the reference instance. 
Table 1: Common configurations of the AV1 encoder

\begin{tabular}{|c|c|c|c|c|c|}
\hline Parameter & Value & Parameter & Value & Parameter & Value \\
\hline cpu-used & 0 & kf-min-dist & 0 & bit-depth & 8 \\
end-usage & $\mathrm{q}$ & kf-max-dist & 9999 & auto-altref & 1 \\
pass & 1 & kf-mode & 1 & drop-frame & 0 \\
\hline
\end{tabular}

\section{Block Structures in AV1}

We introduce some terms that will be used throughout this paper. The depth of a $w \times h$ block is defined to be $\min \left(\log _{2} 64 / w, \log _{2} 64 / h\right)$, i.e. the difference between the base 2 logarithms of 64 and the length of the longer edge. A block is prime if it is not split in the optimal block structure. Two blocks overlap if they have non-empty intersection, regardless of their parent frames. A block $B$ has a split degree of $d_{f}$ in frame $f$, if $d_{f}$ is the maximum depth of the prime blocks in frame $f$ that overlap with $B$. The remote frame of a block $B$ refers to the frame in the reference instance that is identical to the parent frame of $B$.

In AV1, the block structure optimization starts from $64 \times 64$ blocks. A $2 \mathrm{~N} \times 2 \mathrm{~N}$ block can be partitioned into four $\mathrm{N} \times \mathrm{N}$ blocks (referred to as 4-split), two $\mathrm{N} \times 2 \mathrm{~N}$ blocks, two $2 \mathrm{~N} \times \mathrm{N}$ blocks, or no partition at all. A non-square block does not split. Each frame is associated with a q-index that indicates the position of the frame's base quantization parameter (QP) in the lookup table. Periodically some special frames are selected for long-term reference and are given relatively lower QP's, namely intraframes, golden-frames, and altref-frames, to improve quality.

\section{Common Test Settings}

In this paper we use the AV1 codec v0.1.0 [20] running on Ubuntu 15.04 with a 2.40 GHz Intel Xeon E5-2695v2 CPU and 64GB RAM. The common configuration that will be used throughout this paper is given in Table 1. A set of eight test sequences with different spatial resolutions was selected (see Table 2). RD performance and encoding time is measured by BD-rate [21][22] and CPU time.

\section{Statistical Behavior}

Fig. 1(a) shows the percentage of total area for prime blocks as a function of their max depths, averaged across all non-special frames with identical q-indices. Note that we use q-indices rather than the actual QP's for better regression. 50 frames of the sequence BasketballDrill were tested with quality levels set to even values from 22 to 40. Observe that prime blocks in frames with larger q-indices tend to have smaller depths, in other words, partitions occur less often, as expected.

The linear approximations, shown as dotted lines in the figure, give the prior probability of 4-splitting a square block. Denote the linear approximations as $a_{d}(q), d=$ $1, \ldots, 4$ that map q-indices to area percentages. For a square block $B$ in frame $f$ whose depth is $d$, let $d_{f^{\prime}}$ be the split degree of $B$ in an arbitrary frame $f^{\prime}$ with q-index $q$. 


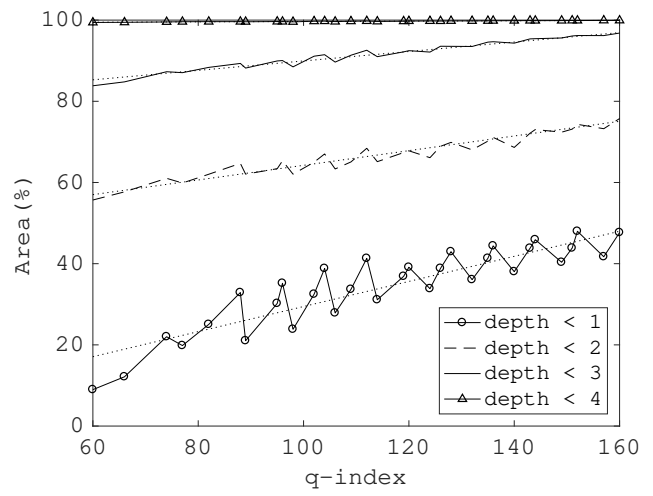

(a)

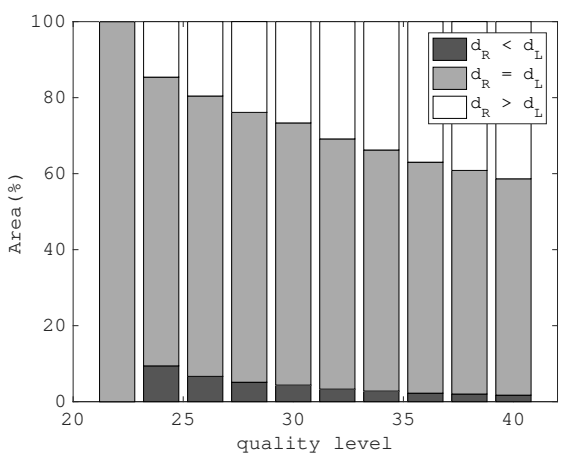

(b)

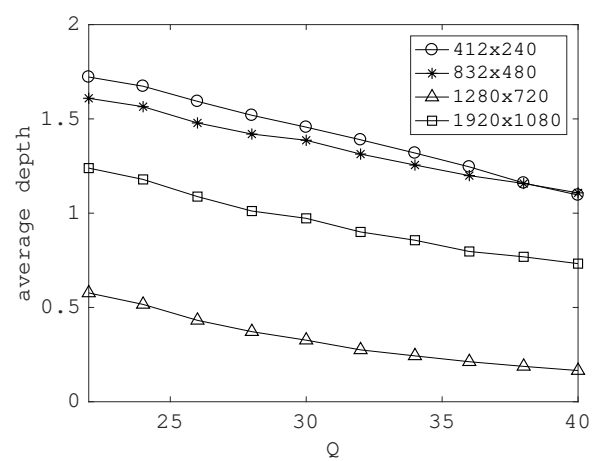

(c)

Figure 1: Statistical behavior of optimal block structures

We roughly estimate $P\left(d_{f^{\prime}}<d\right)$ with $a_{d}(q)$, by the definition of $d_{f^{\prime}}$, we have

$$
P\left(d_{f^{\prime}}>d \mid d_{f^{\prime}}>d-1\right)= \begin{cases}\frac{1-a_{d+1}(q)}{1-a_{d}(q)} & \text { if } d>0 \\ 1-a_{d+1}(q) & \text { if } d=0 .\end{cases}
$$

Now let $f^{\prime}=f$. We see that $d_{f^{\prime}}=d_{f} \geq d$, thus (1) is exactly the probability of $B$ 4-splitting. We will see later that this prior, although established using a single sequence, is robust and applicable to various different sequences for the purpose of this paper.

We now turn to the correlation of optimal block structures between the reference and local instances. Using the same test settings in Fig. 1(a), the instance with the best $Q=22$ is selected as the reference instance. Let $d_{L}$ be the depth of an arbitrary prime block $B$ in frame $f_{L}$ of a local instance, $f_{R}$ be the remote frame of $B$, and $d_{R}$ be the split degree of $B$ in frame $f_{R}$. Fig. 1(b) shows the percentage in total area of prime blocks that satisfy $d_{R}<d_{L}, d_{R}=d_{L}$ or $d_{R}>d_{L}$, averaged across all encoder instances with identical Q's. As expected, when $Q=22$ all blocks have $d_{R}=d_{L}$, since the local instance is identical to the reference instance. The proportion of the case $d_{R}<d_{L}$ is always below $10 \%$, and is even lower as $Q$ increases, while other cases are of notable proportions. This confirms a result for HEVC as reported in [7]. 
Table 2: Experimental results of the RDO pruning algorithms

\begin{tabular}{|l|c|c|c|c|}
\hline \multirow{2}{*}{ Sequence } & \multicolumn{2}{|c|}{ BD-rate } & \multicolumn{2}{c|}{$\Delta T$} \\
\cline { 2 - 5 } & original & improved & original & improved \\
\hline BasketballPass $(412 \times 240)$ & $2.47 \%$ & $0.15 \%$ & $-31.3 \%$ & $-18.9 \%$ \\
BlowingBubbles $(412 \times 240)$ & $3.80 \%$ & $0.25 \%$ & $-32.3 \%$ & $-21.1 \%$ \\
BQSquare $(412 \times 240)$ & $2.04 \%$ & $0.05 \%$ & $-27.2 \%$ & $-17.6 \%$ \\
\hline BasketballDrill $(832 \times 480)$ & $3.75 \%$ & $0.16 \%$ & $-47.8 \%$ & $-30.6 \%$ \\
PartyScene $(832 \times 480)$ & $2.32 \%$ & $0.08 \%$ & $-30.5 \%$ & $-19.8 \%$ \\
\hline FourPeople $(1280 \times 720)$ & $4.09 \%$ & $0.10 \%$ & $-58.6 \%$ & $-40.2 \%$ \\
Johnny $(1280 \times 720)$ & $4.13 \%$ & $0.09 \%$ & $-60.9 \%$ & $-42.4 \%$ \\
\hline Kimono $(1920 \times 1080)$ & $1.61 \%$ & $0.24 \%$ & $-50.9 \%$ & $-37.5 \%$ \\
\hline Average & $\mathbf{3 . 0 3 \%}$ & $\mathbf{0 . 1 4 \%}$ & $\mathbf{- 4 2 . 4 \%}$ & $\mathbf{- 2 8 . 5 \%}$ \\
\hline
\end{tabular}

For future reference, the average depths of prime blocks for each Q and each spatial resolution are also recorded and shown in Fig. 1(c).

\section{Improved RDO Pruning}

We showed in the previous section that by choosing the instance with the best quality as the reference instance, the depth of a prime block rarely exceeds its split degree in its remote frame. [7] proposed a multi-rate encoding algorithm based on a similar observation in HEVC. Specifically, when the depth of a block has reached its split degree in its remote frame, the local instance executes early termination, i.e. 4 -split is not searched in RDO for the current block, as it is unlikely to be the optimal choice. We implemented this algorithm in the AV1 codec, whose results are shown in Table 2 as original. 200 frames of each test sequence were run with $Q=22,27,32,37,42$, choosing $Q=22$ as the reference instance. The time saving is measured by the average time saving of all local instances, with the time cost of the reference instance excluded in the calculations so as to avoid strong dependency on the number of local instances. This is reasonable as the time cost of a single reference instance will become insignificant when the number of local instances is sufficiently large.

[7] reported a time saving of $46 \%$, and a $0.62 \%$ BD-rate on BasketballPass, a significant better tradeoff than our results for AV1. We believe that the difference resulted from the structural difference between AV1 and HEVC on the frame level. The special frames in AV1 play a major role in improving its coding efficiency, as shown in our next experiment. When skipping part of the RDO recursion tree and opting for suboptimal decisions, the overall coding efficiency severely deteriorated. To mitigate this problem, we propose to improve the algorithm by fully encoding special frames in local instances. Using the same settings specified above, the results are shown in Table 2 as improved. As a trade-off between speed and quality, the coding efficiency in terms of BD-rate is much more plausible.

\section{A Bayesian Inference Model}

The last experiment has demonstrated the necessity for keeping block structures of special frames optimal when conducting expedited AV1 encoding. As we run 
exhaustive search on special frames, their optimal block structures become reliable evidence for inference, which will be thoroughly discussed in this section.

Let us assume that $f$ is a non-special frame in a local instance $L, B$ is a block in $f$ that has a depth $d$ and can be 4 -split, $f_{L}$ is the latest special frame in $L$ preceding $f$, and $f_{R}$ is the remote frame of $B$. Let $d_{f^{\prime}}$ denote the split degree of $B$ in any arbitrary frame $f^{\prime}$. Since $f_{L}$ and $f_{R}$ are both fully encoded, $d_{f_{L}}$ and $d_{f_{R}}$ are available.

The key in our multi-rate encoding algorithm is estimating the probability that $B$ is 4 -split in the optimal block structure, knowing $d_{f_{L}}$ and $d_{f_{R}}$, i.e. $P\left(d_{f}>d \mid d_{f_{L}}, d_{f_{R}}\right)$, as $f, f_{L}$ and $f_{R}$ exhibit strong similarities. Since (1) gives the prior probability $p_{0}=P\left(d_{f}>d\right)$, by way of Bayes' theorem

$$
P\left(d_{f}>d \mid d_{f_{L}}, d_{f_{R}}\right)=\frac{P\left(d_{f_{L}}, d_{f_{R}} \mid d_{f}>d\right) p_{0}}{P\left(d_{f_{L}}, d_{f_{R}} \mid d_{f}>d\right) p_{0}+P\left(d_{f_{L}}, d_{f_{R}} \mid d_{f}=d\right)\left(1-p_{0}\right)} .
$$

Note that $d_{f} \geq d$ is always true. This bayesian model allows us to incorporate our prior knowledge about $p_{0}$ into inference. For each depth $d$, we create two 2D tables, namely $T_{d}^{+}$and $T_{d}^{-}$, which record the number of occurrences for the pair $\left(d_{f_{L}}, d_{f_{R}}\right)$ when $d_{f}>d$ and $d_{f}=d$, respectively. $P\left(d_{f_{L}}, d_{f_{R}} \mid d_{f}\right)$ is therefore approximated by

$$
P\left(d_{f_{L}}, d_{f_{R}} \mid d_{f}>d\right)=\frac{T_{d}^{+}\left(d_{f_{L}}, d_{f_{R}}\right)}{\sum_{i, j} T_{d}^{+}(i, j)}, P\left(d_{f_{L}}, d_{f_{R}} \mid d_{f}=d\right)=\frac{T_{d}^{-}\left(d_{f_{L}}, d_{f_{R}}\right)}{\sum_{i, j} T_{d}^{-}(i, j)} .
$$

We substitute (3) into (2) to obtain the posterior probability $p$. Given $\tau_{1}, \tau_{2} \in(0,1)$ and a uniform random variable $X \sim \mathcal{U}(0,1)$, the RDO procedure with regard to $B$ is described by the pseudocode. Intuitively, $\tau_{1}$ is a threshold determining if $p$ is sufficiently small, and $\tau_{2}$ is the sampling frequency for running exhaustive search even if $p$ is small. Early termination is not used if $p$ is not sufficiently small. Even when it is used, we shall still occasionally run full encoding to keep the likelihoods unbiased.

To verify that (3) is a good estimation, suppose we have processed $N$ blocks with depth $d$. Notice that $T_{d}^{+}$is updated when $d_{f}>d$, if we further consider $p$ and $X$ (Cf. line 5-9 in the pseudocode), writing $k_{1}=1$ and $k_{2}=\tau_{2}^{-1}$, we have

$$
\begin{aligned}
E\left(T_{d}^{+}\left(d_{f_{L}}, d_{f_{R}}\right)\right) & =N k_{1} P\left(d_{f_{L}}, d_{f_{R}}, d_{f}>d \mid p>\tau_{1}\right) P\left(p>\tau_{1}\right)+ \\
& N k_{2} P\left(d_{f_{L}}, d_{f_{R}}, d_{f}>d \mid p \leq \tau_{1}, X<\tau_{2}\right) P\left(p \leq \tau_{1} \mid X<\tau_{2}\right) P\left(X<\tau_{2}\right) \\
& =N P\left(d_{f_{L}}, d_{f_{R}}, d_{f}>d\right)
\end{aligned}
$$

as $X$ is indepedent and $k_{2} P\left(X<\tau_{2}\right)=1$. Then the first part of (3) holds assuming $N$ is sufficiently large. The second part of (3) can be proved in a similar fashion.

To see how $\tau_{1}, \tau_{2}$ affect the performance, we treat the posterior probability $p$ as a random variable with density $g(p)$. Let $t_{0}$ and $b_{0}$ be the expected time and bitrate cost of fully encoding a block with depth $d$, while the reduced expected time cost with early termination is $t$, and the increased expected bitrate cost is $b$ if it should be 4-split. Assuming independence between $p$, $t$, and $b$, the expected time saving and bitrate cost increment (in percentage) of encoding $N$ blocks with depth $d$ comparing 


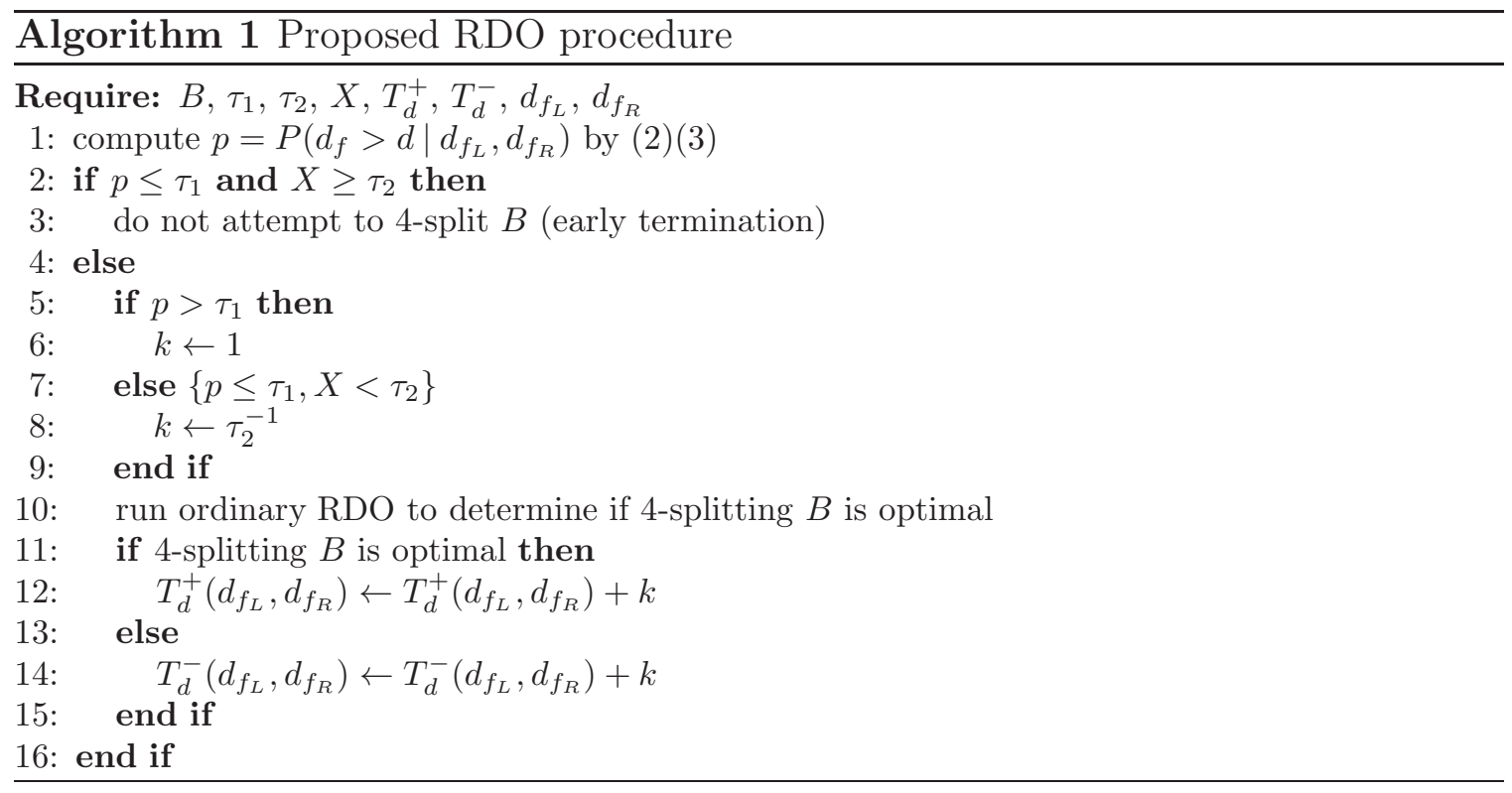

to the original encoder is

$$
\Delta T=\frac{N_{t}\left(t-t_{0}\right)}{N t_{0}}, \Delta B=\frac{N_{b}\left(b-b_{0}\right)}{N b_{0}}
$$

where $N_{t}$ is the expected number of blocks that are not fully encoded, and $N_{b}$ is the expected number of blocks that are not optimally partitioned:

$$
N_{t}=N \int_{0}^{\tau_{1}} g(p)\left(1-\tau_{2}\right) \mathrm{d} p, N_{b}=N \int_{0}^{\tau_{1}} p g(p)\left(1-\tau_{2}\right) \mathrm{d} p<\tau_{1} N_{t}
$$

define $\Delta t=\left(t-t_{0}\right) / t_{0}<0$ and $\Delta b=\left(b-b_{0}\right) / b_{0}>0,(4)(5)$ imply that

$$
\Delta T=\Delta t\left(1-\tau_{2}\right) \int_{0}^{\tau_{1}} g(p) \mathrm{d} p, \frac{\Delta B}{\Delta b}<\tau_{1} \frac{\Delta T}{\Delta t}=\tau_{1}\left(1-\tau_{2}\right) \int_{0}^{\tau_{1}} g(p) \mathrm{d} p .
$$

We see from (6) that a larger $\tau_{1}$ and/or smaller $\tau_{2}$ gives better time saving, and the bitrate penalty is bounded above by the time saving, with a multiplier of $\tau_{1}$. It also implies that a smaller $\tau_{1}$ results in less coding efficiency deterioration.

Finally, to see the effect of $\tau_{2}$ on (3), consider the case $p \leq \tau_{1}$. The increment to $T_{d}^{+}$(or $T_{d}^{-}$) is a Bernoulli random variable $\delta \sim \tau_{2}^{-1} \operatorname{Bern}\left(\tau_{2}\right)$. We see that $E(\delta)=1$ and $\operatorname{Var}(\delta)=\tau_{2}^{-1}-1$. In conclusion, $\tau_{1}, \tau_{2}$ should be chosen carefully to achieve a good balance between time saving, bitrate penalty, and statistical stabilization.

\section{Experimental Results}

Table 3 shows the encoding results of our proposed bayesian method, using the same test setup as Table 2 . We set $\tau_{2}=0.05$ as the analysis in the previous section shows that smaller (but non-zero) $\tau_{2}$ is preferred for better time savings. We varied the value of $\tau_{1}$ to examine its impact on the performances. Fig. 2(a) compares the RD 
Table 3: Experimental results of the bayesian method

\begin{tabular}{|l|c|c|c|c|c|c|}
\hline \multirow{2}{*}{ Sequence } & \multicolumn{3}{|c|}{ BD-rate } & \multicolumn{3}{c|}{$\Delta T$} \\
\cline { 2 - 7 } & $\tau_{1}=0.1$ & $\tau_{1}=0.2$ & $\tau_{1}=0.4$ & $\tau_{1}=0.1$ & $\tau_{1}=0.2$ & $\tau_{1}=0.4$ \\
\hline BasketballPass $(412 \times 240)$ & $0.13 \%$ & $0.20 \%$ & $0.74 \%$ & $-22.5 \%$ & $-24.7 \%$ & $-26.7 \%$ \\
BlowingBubbles $(412 \times 240)$ & $0.16 \%$ & $0.24 \%$ & $0.37 \%$ & $-24.9 \%$ & $-26.3 \%$ & $-29.1 \%$ \\
BQSquare $(412 \times 240)$ & $0.00 \%$ & $0.13 \%$ & $0.46 \%$ & $-18.0 \%$ & $-23.7 \%$ & $-25.7 \%$ \\
\hline BasketballDrill $(832 \times 480)$ & $0.15 \%$ & $0.22 \%$ & $0.58 \%$ & $-30.5 \%$ & $-37.8 \%$ & $-38.4 \%$ \\
PartyScene $(832 \times 480)$ & $0.03 \%$ & $0.20 \%$ & $0.73 \%$ & $-20.2 \%$ & $-24.0 \%$ & $-28.8 \%$ \\
\hline FourPeople $(1280 \times 720)$ & $0.03 \%$ & $0.06 \%$ & $0.07 \%$ & $-18.6 \%$ & $-42.8 \%$ & $-48.2 \%$ \\
Johnny $(1280 \times 720)$ & $0.09 \%$ & $0.10 \%$ & $0.25 \%$ & $-18.8 \%$ & $-37.0 \%$ & $-50.6 \%$ \\
\hline Kimono $(1920 \times 1080)$ & $0.04 \%$ & $0.07 \%$ & $0.14 \%$ & $-10.4 \%$ & $-25.4 \%$ & $-41.5 \%$ \\
\hline Average & $\mathbf{0 . 0 8 \%}$ & $\mathbf{0 . 1 5 \%}$ & $\mathbf{0 . 4 6 \%}$ & $\mathbf{- 2 0 . 5 \%}$ & $\mathbf{- 3 0 . 2 \%}$ & $\mathbf{- 3 6 . 1 \%}$ \\
\hline
\end{tabular}

Table 4: Controlled study of two proposed methods

\begin{tabular}{|l|c|c|c|c|c|}
\hline & & \multicolumn{2}{|c|}{ BD-rate } & \multicolumn{2}{c|}{$\Delta T$} \\
\cline { 3 - 6 } Sequence & $\tau_{1}$ & improved & bayesian & improved & bayesian \\
\hline BasketballPass $(412 \times 240)$ & 0.1 & $0.15 \%$ & $0.13 \%$ & $-18.9 \%$ & $-22.5 \%$ \\
BlowingBubbles $(412 \times 240)$ & 0.1 & $0.25 \%$ & $0.16 \%$ & $-21.1 \%$ & $-24.9 \%$ \\
BQSquare $(412 \times 240)$ & 0.1 & $0.05 \%$ & $0.00 \%$ & $-17.6 \%$ & $-18.0 \%$ \\
\hline BasketballDrill $(832 \times 480)$ & 0.2 & $0.16 \%$ & $0.22 \%$ & $-30.6 \%$ & $-37.8 \%$ \\
PartyScene $(832 \times 480)$ & 0.2 & $0.08 \%$ & $0.20 \%$ & $-19.8 \%$ & $-24.0 \%$ \\
\hline FourPeople $(1280 \times 720)$ & 0.4 & $0.10 \%$ & $0.07 \%$ & $-40.2 \%$ & $-48.2 \%$ \\
Johnny $(1280 \times 720)$ & 0.4 & $0.09 \%$ & $0.25 \%$ & $-42.4 \%$ & $-50.6 \%$ \\
\hline Kimono $(1920 \times 1080)$ & 0.4 & $0.24 \%$ & $0.14 \%$ & $-37.5 \%$ & $-41.5 \%$ \\
\hline \multicolumn{2}{|c|}{ Average } & $\mathbf{0 . 1 4 \%}$ & $\mathbf{0 . 1 4 \%}$ & $\mathbf{- 2 8 . 5 \%}$ & $\mathbf{- 3 3 . 4 \%}$ \\
\hline
\end{tabular}

performances of the original pruning algorithm and our bayesian method regarding the sequence BasketballDrill where $\tau_{1}$ is set to 0.2 .

We see from the results that our proposed method achieves significant time savings (up to 50.6\%) without substantial degradation to coding efficiencies. An interesting pattern is that, sequences with higher spatial resolution can tolerate a larger $\tau_{1}$ in terms of BD-rate cost, in exchange of a significant margin in time savings, while for sequences with lower spatial resolutions this no longer holds, as a smaller $\tau_{1}$ is clearly more favorable. This can be explained by the depths of prime blocks in high resolution sequences being relatively lower (Cf. Fig. 1(c)), encouraging early terminations in the RDO procedure. Nevertheless, setting a constant $\tau_{1}=0.4$ for all sequences yields an average time saving of $36.1 \%$ (and even higher for high resolution sequences), with an average BD-rate cost of $0.46 \%$, which is negligible for most practical applications.

The bayesian approach offers the flexibility to control the tradeoff between time savings and bitrate cost, as shown in Table 3. Furthermore, Table 4 shows the advantage of the joint inference model over the improved pruning method, for controlling the BD-rate cost. Larger $\tau_{1}$ values are chosen for higher resolution sequences, as explained in the previous paragraph. In many cases both BD-rate costs and time savings are improved, without having to trade one for another, and on average time saving can be achieved without BD-rate loss. 


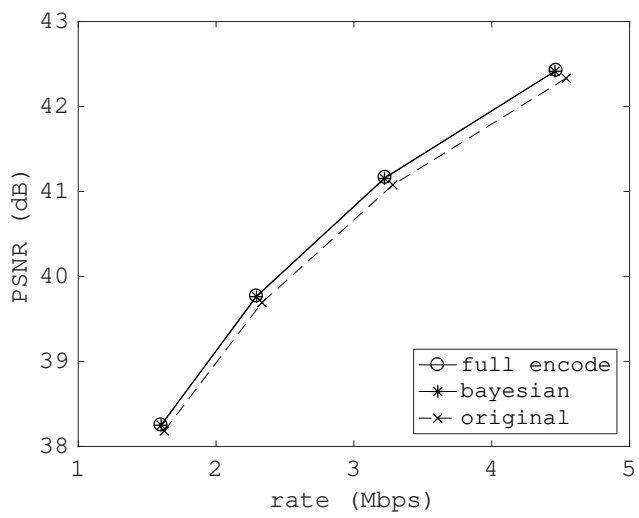

(a)

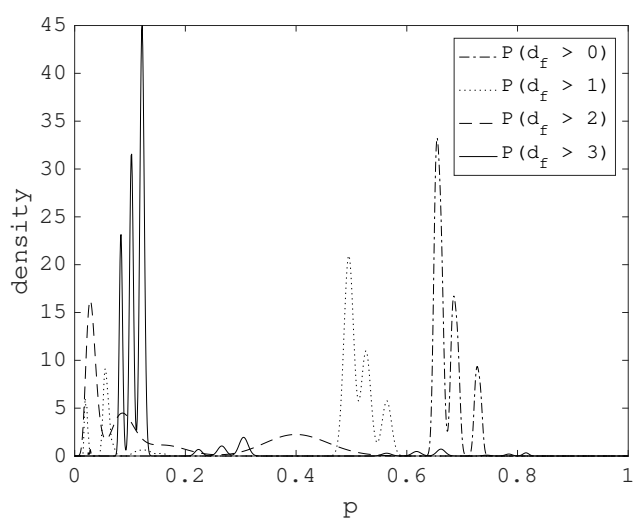

(b)

Figure 2: RD curves and the posterior distribution

Finally, we briefly discuss the statistical behavior of the posterior, previously defined as $g(p)$. Fig. 2(b) shows an estimated $g(p)$ when encoding BasketballDrill under $Q=32$. The bayesian method essentially classifies blocks according to their computed posteriors. According to (6), the portion of $g(p)$ where $p<\tau_{1}$ is eligible for RDO early termination. This implies that a posterior admitting bimodal distribution is ideal for our purpose. Many aspects can be incorporated into the posterior model for further optimization, including spatial resolution, quality levels, RD information, etc, which however, is beyond the scope of this paper.

\section{Conclusions}

In this paper, we propose a novel bayesian block structure inference framework inspired by a modification to an existing HEVC-based multi-rate encoding algorithm. The proposed method is effective, flexible and especially suitable for codecs utilizing special reference frames like AV1. Experimental results show that the proposed bayesian algorithm can achieve $36.1 \%$ time savings in average and up to $50.6 \%$, while keeping the bitrate cost below negligible level.

Future work includes optimizations to the posterior model, dynamic adaptation of $\tau_{1}$, adopting the bayesian approach to prediction mode inference, motion vector/intra mode inference, as well as multi-resolution encoding.

\section{Acknowledgements}

This work was supported by the Natural Science Foundation of China (Project Number 61521002).

\section{References}

[1] Cisco. Cisco visual networking index: Forecast and methodology, 20162021. [Online]. Available: https://www.cisco.com/c/en/us/solutions/collateral/ service-provider/visual-networking-index-vni/complete-white-paper-c11-481360.html 
[2] O. Oyman and S. Singh, "Quality of experience for http adaptive streaming services," IEEE Communications Magazine, vol. 50, no. 4, pp. 20-27, April 2012.

[3] Wikipedia. Aomedia video 1. [Online]. Available: https://en.wikipedia.org/wiki/ AOMedia_Video_1

[4] AOM. Av1 codec library. [Online]. Available: https://aomedia.googlesource.com/aom/

[5] D. Schroeder, A. Ilangovan, M. Reisslein, and E. Steinbach, "Efficient multi-rate video encoding for hevc-based adaptive http streaming," IEEE Transactions on Circuits and Systems for Video Technology, vol. PP, no. 99, pp. 1-1, 2017.

[6] J. D. Praeter, A. J. Daz-Honrubia, N. V. Kets, G. V. Wallendael, J. D. Cock, P. Lambert, and R. V. de Walle, "Fast simultaneous video encoder for adaptive streaming," in 2015 IEEE 17th International Workshop on Multimedia Signal Processing (MMSP), Oct 2015, pp. 1-6.

[7] D. Schroeder, P. Rehm, and E. Steinbach, "Block structure reuse for multi-rate high efficiency video coding," in 2015 IEEE International Conference on Image Processing (ICIP), Sept 2015, pp. 3972-3976.

[8] Z. Zhao and P. Liang, "A highly efficient parallel algorithm for h.264 video encoder," in 2006 IEEE International Conference on Acoustics Speech and Signal Processing Proceedings, vol. 5, May 2006, pp. V-V.

[9] — , "Data partition for wavefront parallelization of h.264 video encoder," in 2006 IEEE International Symposium on Circuits and Systems, May 2006, pp. 4 pp.-2672.

[10] VideoLAN. x264. [Online]. Available: https://www.videolan.org/developers/x264.html

[11] Z. Wen, B. Guo, J. Liu, J. Li, Y. Lu, and J. Wen, "Novel 3d-wpp algorithms for parallel hevc encoding," in 2016 IEEE International Conference on Acoustics, Speech and Signal Processing (ICASSP), March 2016, pp. 1471-1475.

[12] T. Shen, Y. Lu, Z. Wen, L. Zou, Y. Chen, and J. Wen, "Ultra fast h.264/avc to hevc transcoder," in 2013 Data Compression Conference, March 2013, pp. 241-250.

[13] Y. Chen, Z. Wen, J. Wen, M. Tang, and P. Tao, "Efficient software h.264/avc to hevc transcoding on distributed multicore processors," IEEE Transactions on Circuits and Systems for Video Technology, vol. 25, no. 8, pp. 1423-1434, Aug 2015.

[14] I. Ahmad, X. Wei, Y. Sun, and Y.-Q. Zhang, "Video transcoding: an overview of various techniques and research issues," IEEE Transactions on Multimedia, vol. 7, no. 5, pp. 793-804, Oct 2005.

[15] B. Li and J. Liu, "Multirate video multicast over the internet: an overview," IEEE Network, vol. 17, no. 1, pp. 24-29, Jan 2003.

[16] D. H. Finstad, H. K. Stensland, H. Espeland, and P. Halvorsen, "Improved multi-rate video encoding," in 2011 IEEE International Symposium on Multimedia, Dec 2011, pp. 293-300.

[17] Q. Hu, Z. Shi, X. Zhang, and Z. Gao, "Early skip mode decision based on bayesian model for hevc," in 2015 Visual Communications and Image Processing (VCIP), Dec 2015, pp. 1-4.

[18] X. Shen, L. Yu, and J. Chen, "Fast coding unit size selection for hevc based on bayesian decision rule," in 2012 Picture Coding Symposium, May 2012, pp. 453-456.

[19] C. Cai, S. Yin, X. Zhang, and Z. Gao, "An efficient hevc multi-rate encoding system based on x265," in 2016 Visual Communications and Image Processing (VCIP), Nov 2016, pp. 1-4.

[20] AOM. v0.1.0, aom, git at google. [Online]. Available: https://aomedia.googlesource. $\mathrm{com} / \mathrm{aom} /+\mathrm{v} 0.1 .0$

[21] G.Bjontegaard, "Calculation of average psnr differences between rd curves," in Doc. VCEG-M33 ITU-T Q6/16, 2001.

$[22]-$ - "Improvements of the bd-psnr model," in ITU-T SG16 Q, vol. 6, 2008, p. 35. 